\title{
Phytoprotection
}

\section{Abondance saisonnière des espèces de pyrales des prés (Lepidoptera : Crambidae) associées aux graminées à gazon au Québec}

\section{Seasonal abundance of the sod webworm species (Lepidoptera: Crambidae) associated with turfgrass in Quebec}

\section{Sophie Rochefort, Yves Carrière, David J. Shetlar et Jacques Brodeur}

Volume 84, numéro 2, août 2003

$\mathrm{V}^{\mathrm{e}}$ Conférence internationale francophone d'entomologie. « La recherche de pointe en entomologie ". Montréal (Québec), Canada, 14-18 juillet 2002

URI : https://id.erudit.org/iderudit/007809ar DOI : https://doi.org/10.7202/007809ar

Aller au sommaire du numéro

Éditeur(s)

Société de protection des plantes du Québec (SPPQ)

ISSN

0031-9511 (imprimé)

1710-1603 (numérique)

Découvrir la revue

Citer cet article

Rochefort, S., Carrière, Y., Shetlar, D. J. \& Brodeur, J. (2003). Abondance saisonnière des espèces de pyrales des prés (Lepidoptera: Crambidae) associées aux graminées à gazon au Québec. Phytoprotection, 84(2), 69-75. https://doi.org/10.7202/007809ar
Résumé de l'article

L'échantillonnage de 15 terrains gazonnés des régions de Québec et de Montréal, Québec, Canada, a permis d'identifier les espèces de pyrales des prés présentes dans les pelouses urbaines, ainsi que celles causant des dommages au stade larvaire. Le dépistage des papillons a été réalisé de juin à septembre à l'aide de pièges lumineux, et celui des larves à l'aide d'un perce-trou de golf (emporte-pièce). Onze espèces de pyrales des prés adultes ont été capturées dans les pièges lumineux en 1998 et quatre en 1999. De ces espèces, trois étaient plus abondantes et associées aux pelouses, soit Crambus praefectellus, Pediasia trisecta, la grande pyrale des prés, et Chrysoteuchia topiaria, l'anneleur de la canneberge. Les patrons d'abondance saisonnière des adultes de ces trois espèces durant la saison estivale ont été établis pour 1998 et 1999. L'anneleur de la canneberge est la seule espèce qui a causé des dommages aux pelouses étudiées. 


\title{
Abondance saisonnière des espèces de pyrales des prés (Lepidoptera : Crambidae) associées aux graminées à gazon au Québec
}

\author{
Sophie Rochefort ${ }^{1}$, Yves Carrière ${ }^{2}$, David J. Shetlar ${ }^{3}$ et Jac- \\ ques Brodeur ${ }^{1}$
}

Reçu 2002-08-15; accepté 2003-03-11

\section{PHYTOPROTECTION $84: 69-75$}

L'échantillonnage de 15 terrains gazonnés des régions de Québec et de Montréal, Québec, Canada, a permis d'identifier les espèces de pyrales des prés présentes dans les pelouses urbaines, ainsi que celles causant des dommages au stade larvaire. Le dépistage des papillons a été réalisé de juin à septembre à l'aide de pièges lumineux, et celui des larves à l'aide d'un perce-trou de golf (emporte-pièce). Onze espèces de pyrales des prés adultes ont été capturées dans les pièges lumineux en 1998 et quatre en 1999. De ces espèces, trois étaient plus abondantes et associées aux pelouses, soit Crambus praefectellus, Pediasia trisecta, la grande pyrale des prés, et Chrysoteuchia topiaria, I'anneleur de la canneberge. Les patrons d'abondance saisonnière des adultes de ces trois espèces durant la saison estivale ont été établis pour 1998 et 1999. L'anneleur de la canneberge est la seule espèce qui a causé des dommages aux pelouses étudiées.

[Seasonal abundance of the sod webworm species (Lepidoptera: Crambidae) associated with turfgrass in Quebec]

Fifteen lawns in Quebec City and Montreal, Quebec, Canada, were sampled in 1998 and 1999 to establish the sod webworm species complex associated with turfgrass and to determine the damaging species at the larval stage. Sod webworm adults were captured with light traps from June to September. A golf cup cutter served to monitor larvae in sod with webworm damage. Eleven sod webworm species were captured with light traps in 1998 and four in 1999. Three of these species were dominant: Crambus preafectellus (silver-striped webworm), Pediasia trisecta (larger sod webworm) and Chrysoteuchia topiaria (cranberry girdler). The seasonal abundance of these species at the adult stage was established during the summer of 1998 and 1999. The cranberry girdler was the only species that caused damages to the turf.

1. Centre de Recherche en Horticulture, Université Laval, Pavillon Envirotron, Sainte-Foy, Québec, Canada G1K 7P4. Pour correspondance : jacques.brodeur@plg.ulaval.ca

2. Department of Entomology, University of Arizona, P.O. Box 210106, Tucson, AZ USA 85721-0106

3. Department of Entomology, Ohio State University, 1991 Kenny Road, Columbus, Ohio USA 43210 


\section{INTRODUCTION}

Les pyrales des prés (Lepidoptera : Crambidae) représentent un groupe d'espèces pouvant causer des dommages importants aux pelouses résidentielles et commerciales en milieu urbain (Potter 1998; Tashiro 1987). Les papillons de petites tailles $(19-25 \mathrm{~mm}$ d'envergure d'ailes) sont actifs la nuit et se réfugient le jour au sol ou dans la végétation arbustive. Les larves vivent dans le sol ou le chaume et se nourrissent principalement des racines et de la couronne des graminées à gazon, ce qui entraîne le flétrissement, le jaunissement et la mort de la plante en fin de saison estivale (Tashiro 1987). Toutes les espèces de graminées à gazon sont susceptibles d'être attaquées par les larves de pyrales des prés, en particulier le pâturin du Kentucky, l'espèce dominante des régions froides, l'agrostide et les fétuques fines (Niemczyk et Shetlar 2000). En Amérique du Nord, plus d'une vingtaine d'espèces de pyrales, essentiellement de la famille des Crambidae (Minet 1985; Munroe et Solis 1999) se trouvent sur les pelouses mais, typiquement, deux à trois espèces par région causent des problèmes (Watschke et al. 1995).

Au Québec, les pyrales des prés causent sporadiquement et localement des dommages importants aux pelouses urbaines (Rochefort et al. 1999). L'identité des espèces ainsi que leur cycle saisonnier d'activité sont inconnus. Les objectifs de cette étude étaient d'identifier les espèces de pyrales des prés présentes au Québec, de déterminer celles qui sont les plus dommageables et d'établir les périodes d'activité des papillons. Ces informations s'avèrent essentielles à l'établissement d'une stratégie efficace de lutte à ces ravageurs.

\section{MATÉRIEL ET MÉTHODES}

Durant deux saisons estivales, 1998 et 1999, nous avons échantillonné des larves et des adultes de pyrales des prés dans deux régions du Québec, la région de Montréal $\left(45^{\circ} 31^{\prime} \mathrm{N}, 73^{\circ} 39^{\prime} \mathrm{O}\right)$ et celle de Québec $\left(46^{\circ} 49^{\prime} \mathrm{N}, 71^{\circ} 13^{\prime} \mathrm{O}\right)$.

\section{Adultes}

Les adultes ont été capturés à l'aide de pièges lumineux, LUMINOC $\circledast$ version LC (Biocom, Québec, Canada), munis d'un tube fluorescent vert (longueur $d^{\prime}$ 'onde de 550 nanomètres et puissance de 1,8 watts). Chaque piège était fixé à une tige de métal à un mètre au-dessus du sol, une hauteur déterminée selon des études réalisées sur l'anneleur de la canneberge et autres pyrales des prés (Kamm et al. 1983; Tashiro 1987). Un morceau $(2 \times 5 \mathrm{~cm})$ de plaquette insecticide Vapona ${ }^{\circledR}$ était placé à l'intérieur de chaque piège afin de tuer rapidement les spécimens capturés. Les pièges étaient activés à la brunante à l'aide d'une cellule photoélectrique. Cette dernière assurait le fonctionnement automatique du piège pour une période de $4 \mathrm{~h}$ par le biais d'une minuterie. Cet intervalle correspond à la principale période d'activité des pyrales des prés (Tashiro 1987).

La capture des papillons s'est échelonnée entre le début du mois de juin et le début du mois de septembre; cet intervalle couvre les périodes d'activité de vol des principales espèces de pyrales des prés dans le nord-est des EtatsUnis (Niemczyk et Shetlar 2000). Les pièges ont été vidés une fois par semaine et les spécimens, conservés pour identification. Quinze pièges ont été installés sur des terrains résidentiels et municipaux ayant un historique d'infestation par les pyrales des prés (Rochefort et al. 1999). En 1998, il y avait 11 pièges dans la région de Montréal (Île Bizard, Ville Saint-Laurent, Dollarddes-Ormeaux, Saint-Léonard, Boucherville, Pointe-aux-Trembles, Jardin Botanique de Montréal, Saint-Lambert, Montréal-Nord, Ahunstic, Beloeil) et quatre dans la région de Québec (Charlesbourg, Québec, Sillery, Beauport). En 1999 , il y avait neuf pièges dans la région de Montréal (Beloeil, Boucherville, Varennes, Saint-Bruno-de-Montarville, Rosemère, Deux-Montagnes) et six autres dans la région de Québec (Beauport, Saint-Émile, Neufchâtel, Sillery, Sainte-Foy). II y avait parfois plus d'un piège par ville. 


\section{Larves}

Les larves ont été échantillonnées à I'aide d'un perce-trou de golf (Tashiro 1987). La technique consiste à prélever un échantillon de sol et de gazon à l'aide d'un emporte-pièce $(9 \mathrm{~cm}$ de diam x 30 $\mathrm{cm}$ de profondeur) et de l'examiner attentivement pour dénombrer les larves. Ces dernières ont été conservées dans de l'éthanol $70 \%$ pour identification ultérieure. Six des 15 terrains à l'étude ont été échantillonnés en 1998 et en 1999 aux dates suivantes : les 28 et 29 juillet, le 5 août, les 3 et le 4 septembre et finalement, le 11 septembre. Pour chacune de ces dates, 10 échantillons ont été prélevés dans les zones endommagées de chaque terrain. Ces terrains étaient situés dans la région de Montréal (Boucherville, Dollarddes-Ormeaux, île Bizard, Beloeil).

Les larves ont été identifiées au laboratoire d'entomologie de I'Université d'Ohio selon les caractéristiques morphologiques décrites dans diverses publications (Ainslie 1923a, 1923b, 1927; Kennedy 1980), ainsi qu'à partir d'une collection de référence.

\section{RÉSULTATS}

\section{Adultes}

Pour chacune des dates d'échantillonnage, le nombre total de papillons cap- turés dans les pièges lumineux pour les régions de Montréal et de Québec en 1998 et 1999 est présenté au tableau 1.

Dans la région de Montréal, 11 espèces ont été capturées en 1998 et quatre en 1999. Dans la région de Québec, sept espèces ont été capturées en 1998 et quatre en 1999. Parmi ces espèces, trois étaient plus abondantes: I'anneleur de la canneberge, Chrysoteuchia topiaria Zeller (cranberry girdler), Crambus praefectellus Zincken (silver striped webworm) et la grande pyrale des prés, Pediasia trisecta Walker (larger sod webworm). Selon diverses études réalisées en Amérique du Nord (Tashiro 1987; Tolley et Robinson 1986), ces trois espèces sont associées aux graminées à gazon. Dans la région de Montréal, ces trois espèces représentaient respectivement 84 et $91 \%$ du nombre total d'individus capturés en 1998 et 1999. Dans la région de Québec, elles représentaient respectivement 87 et $75 \%$ des individus échantillonnés en 1998 et 1999. Les autres espèces n'ont pas été identifiées. L'abondance saisonnière des trois espèces dominantes de papillons dans les régions de Montréal et de Québec est présentée aux figures 1 et 2.

En 1998 et 1999, tant dans les régions de Montréal que de Québec, l'espèce dominante était $C$. topiaria. Les adultes étaient présents de la mi-juin à la fin du

Tableau 1. Nombre total de pyrales des prés capturées dans les pièges lumineux des régions de Montréal et de Québec en 1998 et 1999

\begin{tabular}{|c|c|c|c|c|}
\hline \multirow{2}{*}{$\begin{array}{l}\text { Date } \\
\text { d'échantillonnage }\end{array}$} & \multicolumn{2}{|c|}{ Montréal } & \multicolumn{2}{|c|}{ Québec } \\
\hline & 1998 & 1999 & 1998 & 1999 \\
\hline $7 / 06$ & 2 & 4 & 0 & 0 \\
\hline $14 / 06$ & 3 & 2 & 0 & 0 \\
\hline $21 / 06$ & 13 & 13 & 4 & 0 \\
\hline $28 / 06$ & 27 & 24 & 7 & 3 \\
\hline $5 / 07$ & 11 & 25 & 4 & 6 \\
\hline $12 / 07$ & 29 & 16 & 8 & 6 \\
\hline $19 / 07$ & 12 & 34 & 8 & 1 \\
\hline $26 / 07$ & 7 & 14 & 5 & 2 \\
\hline $2 / 08$ & 7 & 10 & 2 & 1 \\
\hline $9 / 08$ & 7 & 10 & 2 & 3 \\
\hline $16 / 08$ & 5 & 10 & 2 & 2 \\
\hline $23 / 08$ & 9 & 13 & 3 & 0 \\
\hline $30 / 08$ & 1 & 12 & 1 & 0 \\
\hline $6 / 09$ & 0 & 13 & 0 & 0 \\
\hline $13 / 09$ & 0 & 8 & 0 & 0 \\
\hline $20 / 09$ & 0 & 0 & 0 & 0 \\
\hline
\end{tabular}




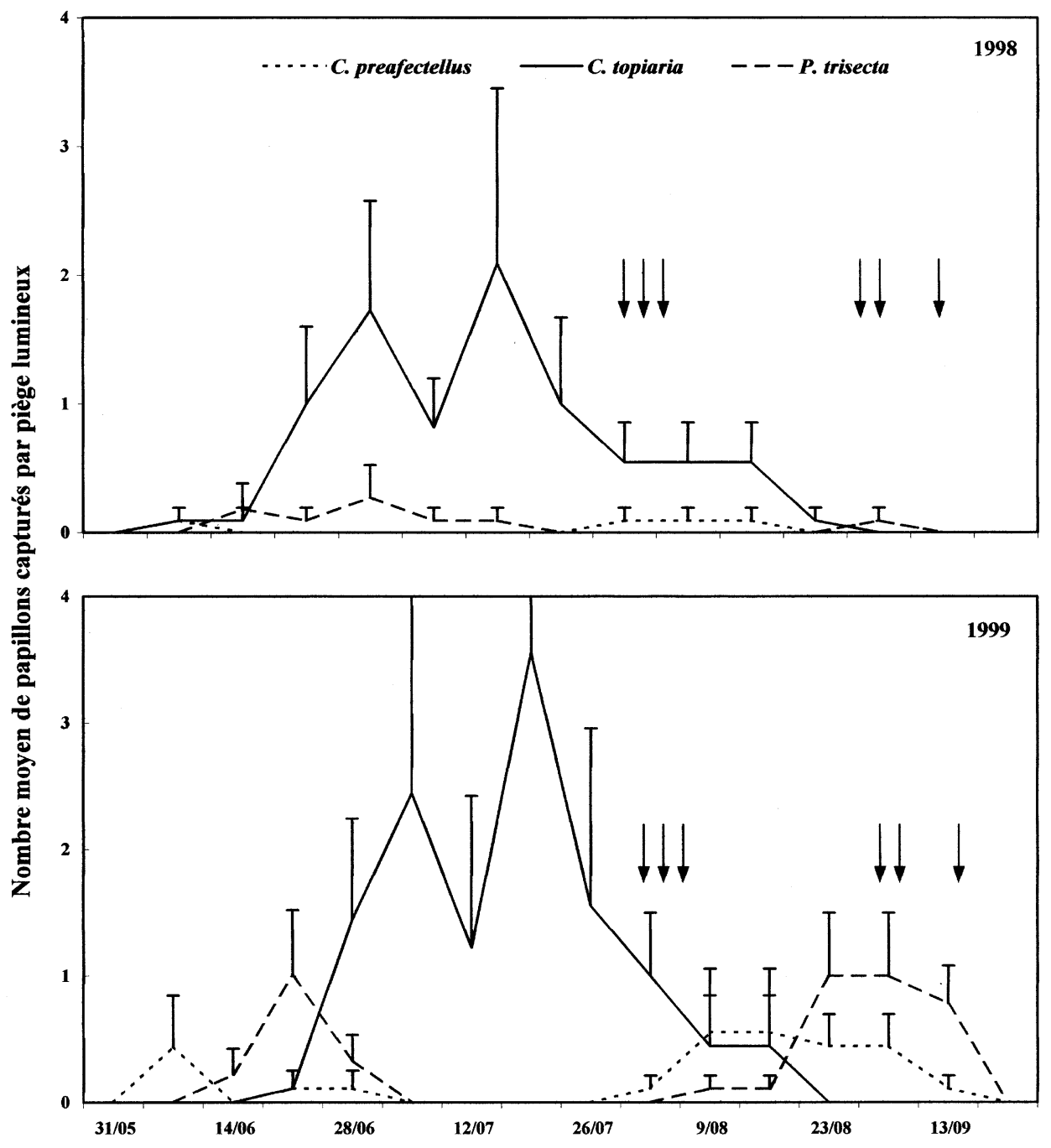

Figure 1. Abondance relative (nombre moyen de spécimens par piège $\pm s_{x}$ ) des trois principales espèces de pyrales des prés adultes capturées dans des pièges lumineux en 1998 et 1999 dans la région de Montréal. Les flèches indiquent les dates d'échantillonnage des larves.

mois d'août. En 1998, le nombre moyen de papillons capturés par piège était similaire pour les deux régions mais les captures de $C$. topiaria étaient plus abondantes dans la région de Montréal que dans celle de Québec en 1999 (Figs. 1 et 2).

Pediasia trisecta a été capturé à Montréal en 1998 et en 1999 (Fig. 1), mais uniquement en 1998 à Québec (Fig.
2). Cette espèce a deux périodes de vol dans la région de Montréal, une première en juin et une seconde à la fin août/début septembre (Fig. 1).

Plusieurs individus de C. praefectellus ont été capturés en 1998 et en 1999 dans la région de Montréal (Fig. 1). L'espèce était aussi présente dans la région de Québec en 1998 (Fig. 2) mais un seul papillon a cependant été captu- 


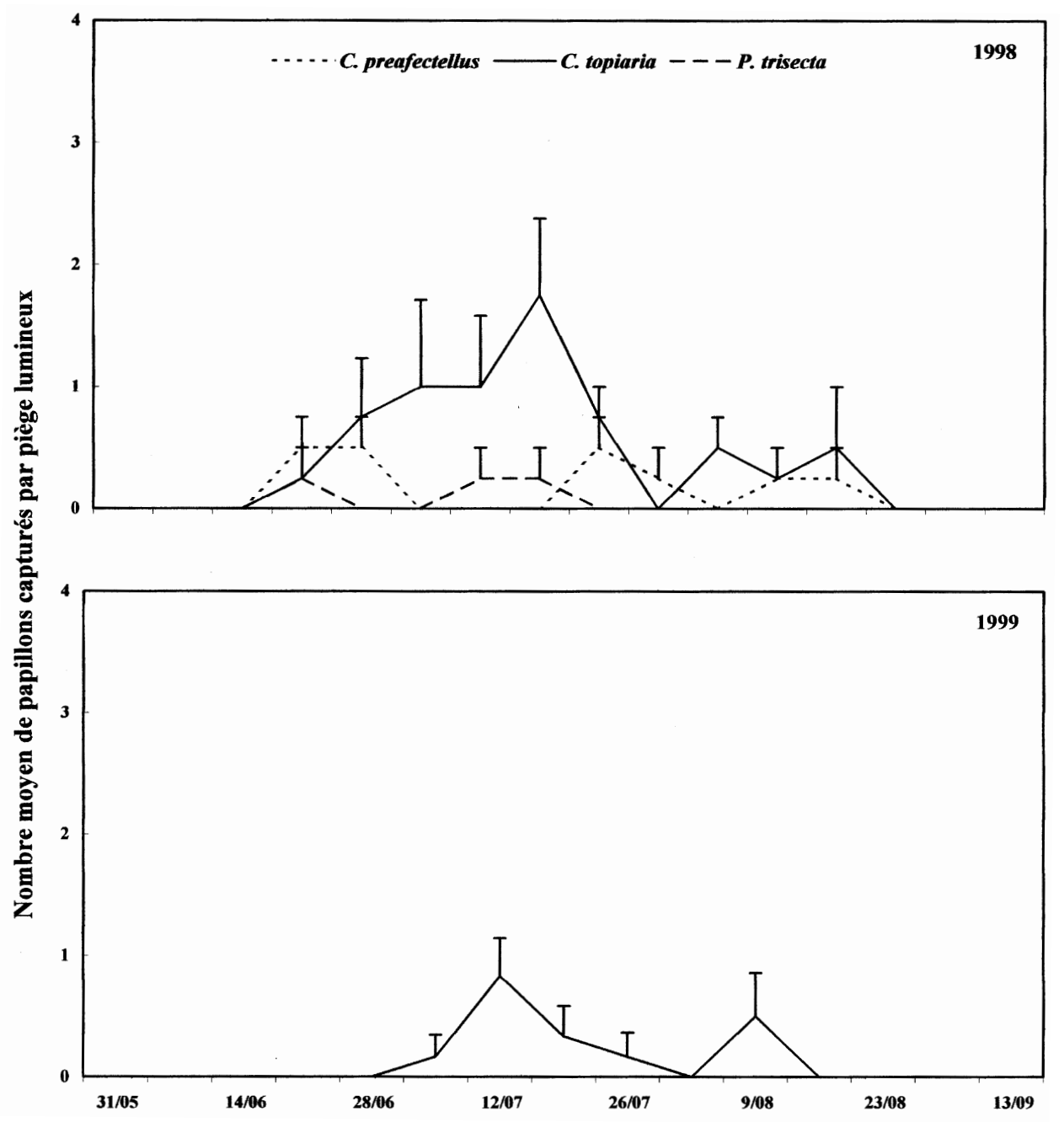

Figure 2. Abondance relative (nombre moyen de spécimens par piège $\pm s_{x}$ ) des trois principales espèces de pyrales des prés adultes capturées dans des pièges lumineux en 1998 et 1999 dans la région de Québec.

ré en 1999 (non-illustré sur la figure 2). Tout comme $P$. trisecta, cette espèce semble avoir deux périodes de vol, une première s'étalant du début jusqu'à la fin du mois de juin et une seconde de la fin juillet à la fin août.

\section{Larves}

La seule espèce de pyrale des prés retrouvée à l'état larvaire dans les pelouses endommagées de la région de Montréal est $C$. topiaria. Des moyennes $\left( \pm s_{x}\right)$ de $0,5 \pm 0,41$ et de $1,4 \pm 0,69$ larves par échantillon ont été observées respectivement en 1998 et 1999. 


\section{DISCUSSION}

Les captures de papillons dans les pièges lumineux et de larves dans les pelouses nous permettent de dresser un premier portrait des pyrales des prés présentes au Québec en milieu urbain. Trois principales espèces de Crambidae ont été observées, dont une seule au stade larvaire, I'anneleur de la canneberge, dans les zones de pelouse endommagée. Seule cette dernière espèce est considérée comme nuisible au Québec (Rochefort et al. 1999). Ce patron diffère sensiblement de ceux rapportés dans le nord-est des États-Unis où l'on retrouve généralement un complexe formé de deux à trois espèces qui causent des dommages aux pelouses (Tashiro 1987). Potter (1998) mentionne que les espèces de pyrales les plus communes s'attaquant aux pelouses des régions de climat froid sont: Parapediasia trisecta (Walker), Fissicrambus mutabilis (Clemens), Pediasia trisecta (Walker) et Crambus praefectellus (Zincken).

Bien que des adultes de $C$. preafectellus et de Pediasia trisecta aient été fréquemment capturés dans les pièges lumineux positionnés au-dessus des pelouses urbaines, aucune larve de ces deux espèces n'a été trouvée dans le gazon. De même, la présence de papillons dans les pièges lumineux ne semble pas être un bon indicateur d'une infestation sévère des pelouses par les larves. Des travaux supplémentaires sont toutefois nécessaires pour établir, s'il y a lieu, une corrélation entre les captures des pièges lumineux et l'intensité des dommages sur les pelouses adjacentes. Nos observations suggèrent toutefois que les activités de dépistage, dans le contexte d'un programme de contrôle de ces ravageurs, doivent essentiellement être orientées vers les larves au niveau du gazon.

Chrysoteuchia topiaria est une pyrale commune des zones tempérées. L'espèce est univoltine sur toute son aire de répartition (Kamm et al. 1983; Tolley et Robinson 1986; Watschke et al. 1995). Les adultes émergent au début de l'été et sont actifs jusqu'à la mi-août. Le développement des larves dure envi- ron 2 mois et elles se nourrissent de la couronne et des racines des graminées à gazon. Les dommages sur les terrains ne sont visibles qu'à l'automne. En octobre, les prépupes tissent un hibernaculum et entrent en diapause dans le sol ou dans le feutre (Watschke et al. 1995). Rochefort et al. (1999) ont identifié certaines caractéristiques des pelouses susceptibles aux infestations par l'anneleur de la canneberge. Ainsi, le ravageur préfèrerait les jeunes pelouses de pâturin du Kentucky, généralement âgées de moins de 10 ans, les sites avec un degré d'ensoleillement important et les pelouses qui présentent un problème d'épaisseur de feutre $(>15$ $\mathrm{mm})$. Selon Watschke et al. (1995), I'anneleur de la canneberge s'observe principalement sur des terrains constitués de pâturin du Kentucky, d'agrostide et de fétuque fine où les jeunes larves colonisent le feutre de la pelouse plutôt que le sol, cela afin d'être à proximité de la couronne des plantes. Ceci pourrait expliquer pourquoi les jeunes pelouses ayant un feutre épais sont davantage endommagées par I'anneleur de la canneberge.

La grande pyrale des prés, $P$. trisecta est un ravageur important des pelouses dans les zones tempérées des ÉtatsUnis (Potter 1998; Tashiro 1987). Nos résultats indiquent qu'elle est présente dans les régions de Montréal et de Québec mais ne cause pas de dommages importants aux pelouses urbaines. L'espèce montre une phénologie saisonnière particulière puisqu'elle est univoltine dans l'ouest du continent mais multivoltine à l'est des montagnes Rocheuses (Niemczyk et Shetlard 2000). On observe deux générations par année en Ohio et en lowa (Ainslie 1927) et trois générations au New Jersey (Mailloux et Streu 1982). Au Québec, l'espèce est bivoltine.

Crambus preafectellus est un ravageur commun des pelouses sur toute la côte est américaine (Tashiro 1987). II existe très peu d'information sur son cycle saisonnier. Selon Ainslie (1923), I'espèce est trivoltine aux États-Unis et serait la première à être observée sur les pelouses au printemps. Nos données sont préliminaires mais suggèrent 
que $C$. preafectellus complète deux générations par année au Québec et que les adultes sont effectivement actifs tôt en début de saison.

Au cours des dernières décennies, I'utilisation d'insecticides à large spectre sur les pelouses urbaines a contribué à accentuer les dommages causés par les pyrales des prés en éliminant leurs ennemis naturels (Cockfield et Potter 1984). Au Québec, on constate des infestations de plus en plus importantes de l'anneleur de la canneberge dans les régions de Montréal (municipalités de Terrebonne, Beloeil, Bois-desFilion) et de Québec (municipalités de Charlesbourg, Beauport) (Rochefort et al. 1999). Outre certaines pratiques culturales (irrigation, fertilisation et élimination des excès de chaume), les nématodes entomopathogènes présentent un bon potentiel pour le contrôle des pyrales des prés dans les pelouses urbaines (Simard et al. 2002).

\section{REMERCIEMENTS}

Cette étude a été réalisée grâce au support financier de I'Association des services en horticulture ornementale du Québec et du ministère de l'Environnement du Québec. Nous remercions également l'Agence canadienne d'inspection des aliments pour le prêt des pièges lumineux, divers citoyens et les municipalités de Montréal et de SaintLaurent pour leur participation à cette étude, ainsi que deux réviseurs anonymes pour leurs commentaires constructifs sur ce manuscrit.

\section{RÉFÉRENCES}

Ainslie, G.G. 1923a. Silver-striped webworm, Crambus praefectellus, Zincken. J. Agric. Res. 24 : 415-426.

Ainslie, G.G. 1923b. Striped webworm, Crambus mutabalis, Clemens. J. Agric. Res. 24 : 399-414.

Ainslie, G.G. 1927. The larger sod webworm. US Dept. Agric. Tech. Bull. No. 31. 17 pp.

Cockfield, S.D. et D.A. Potter. 1984. Predation on sod webworm (Lepidoptera : Pyralidae) eggs as affected by Chlorpyrifos applications to Kentucky Bluegrass turf. J. Econ. Entomol. 77 : 1542-1544.
Kamm, J.A., P.D. Morgan, D.L. Overhulser, L.M. McDonough, M. Triebwasser et L.N. Kline. 1983. Management practices for cranberry girdler (Lepidoptera : Pyralidae) in Douglas-fir nursery stock. J. Econ. Entomol. 76 : 923-926.

Kennedy, M.K. 1980. New webworm pests in Michigan lawns. Amer. Lawn Appl., Nov./ Déc.: 11-14.

Mailloux, G. et H.T. Streu. 1982. Bionomics of the larger sod webworm, Pediasia trisecta (Walker) (Lepidoptera : Pyralidae, Crambinae). Ann. Soc. Entomol. Québec $27: 68-74$.

Minet, J. 1985. Étude morphologique et phylogénétique des organes tympaniques des Pyraloidea. 2. Pyralidae, Crambidae, première partie (Lepidoptera Glossata). Ann. Soc. Entomol. Fr. 21 : 69-86.

Munroe, E.G. et M.A. Solis. 1999. The Pyraloidea. Pages 233-256 in N.P. Kristensen (réd.), Lepidoptera, Moths and Butterflies. Volume 1 : Evolution, Systematics, and Biogeography. Handbook of Zoology. Walter de Gruyter, Berlin et New York.

Niemczyk, H.D. et D.J. Shetlar. 2000. Destructive turf insects. $2^{\mathrm{e}}$ éd. H.D.N. Books, Wooster, Ohio. 148 pp.

Potter, D.A. 1998. Destructive turfgrass insects. Ann Arbor Press, Chelsea, MI. 344 pp.

Rochefort, S., J. Brodeur, Y. Desjardins et Y. Carrière. 1999. Projet-pilote de lutte intégrée dans les pelouses. Rapport de recherche présenté à I'Association des services en horticulture ornementale du Québec. Université Laval, Québec (Québec). 94 pp.

Simard, L., G. Bélair et J. Brodeur. 2002. Susceptibility of the cranberry girdler to entomopathogenic nematodes. Can. Entomol. $134: 329-330$.

Tashiro, H. 1987. Turfgrass insects of the United States and Canada. Cornell University Press, Ithaca, NY. 391 pp.

Tolley, M.P. et W.H. Robinson. 1986. Seasonal abundance and degree-day prediction of sod webworm (Lepidoptera : Pyralidae) adult emergence in Virginia. J. Econ. Entomol. 79 : 400-404.

Watschke, T.L., P.H. Dernoeden et D.J. Shetlar. 1995. Managing turfgrass pests. Lewis Publishers, Boca Raton, FL. 361 pp. 